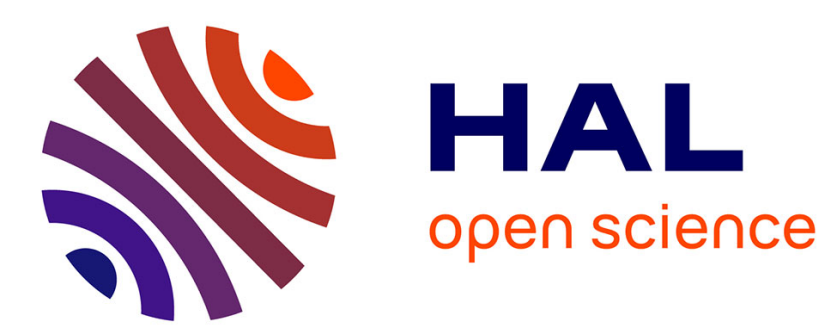

\title{
Velocity-free attitude stabilization with inertial vector measurements
}

Lotfi Benziane, Abdelaziz Benallegue, Yacine Chitour, Abdelhamid Tayebi

\section{To cite this version:}

Lotfi Benziane, Abdelaziz Benallegue, Yacine Chitour, Abdelhamid Tayebi. Velocity-free attitude stabilization with inertial vector measurements. International Journal of Robust and Nonlinear Control, 2016, 26 (11), pp.2478-2493. 10.1002/rnc.3407 . hal-02307516

\section{HAL Id: hal-02307516 \\ https://hal-centralesupelec.archives-ouvertes.fr/hal-02307516}

Submitted on 11 Apr 2020

HAL is a multi-disciplinary open access archive for the deposit and dissemination of scientific research documents, whether they are published or not. The documents may come from teaching and research institutions in France or abroad, or from public or private research centers.
L'archive ouverte pluridisciplinaire HAL, est destinée au dépôt et à la diffusion de documents scientifiques de niveau recherche, publiés ou non, émanant des établissements d'enseignement et de recherche français ou étrangers, des laboratoires publics ou privés. 


\title{
Velocity-free Attitude Stabilization with Inertial Vector Measurements
}

\author{
L. Benziane*, A. Benallegue ${ }^{\ddagger}$, Y. Chitour ${ }^{\S}$, A. Tayebi ${ }^{\Uparrow /}$ \\ *,+ LISV, Université de Versailles Saint Quentin en Yvelines, France. \\ ${ }^{\S}$ L2S, Université Paris-Sud XI, CNRS and Supélec, Gif-sur-Yvette, and Team GECO, INRIA Saclay, France. \\ II Department of Electrical Engineering, Lakehead University, Thunder Bay, Ontario, Canada.
}

\begin{abstract}
SUMMARY
This paper deals with the attitude stabilization problem of a rigid body, where neither the angular velocity nor the attitude are used in the feedback; only body-referenced vector measurements are needed. The proposed control scheme is based on an angular velocity observer-like system relying solely on vector measurements. The proposed controller ensures almost global asymptotic stability and provides some interesting performance properties through an appropriate tuning of the control gains. The performance and effectiveness of the proposed control scheme are illustrated via simulation results where the control gains are adjusted using a nonlinear optimization. Copyright (c) 0000 John Wiley \& Sons, Ltd.

Received ...
\end{abstract}

KEY WORDS: Attitude; Stabilization; AGAS.

\section{INTRODUCTION}

The stabilization of a rotational motion of a rigid body is a well studied problem in the literature (see, for instance, [1-7]). Despite the large number of existing solutions to this problem, it remains an active research topic due to its challenges and potential applications in many fields such as robotics, unmanned aerial vehicles (UAVs), satellites, marine vehicles, etc. The attitude control problem has been dealt with in the literature using different types of attitude parametrization such as the Euler angles and the modified Rodrigues parameters which are local and minimal attitude representations (see, for instance, $[8,9])$. Other solutions relying on the global and unique attitude representation evolving in the Special Orthogonal group $\mathrm{SO}(3)$ have also been proposed in the literature (see, for instance, $[10,11])$. However, for the sake of design and implementation simplicity, a number of

${ }^{*}$ Correspondence to: Lotfi BENZIANE, Laboratoire d'ingénierie des Systèmes de Versailles, 10-12 avenue de 1' Europe, 78140 Vélizy, FRANCE. Email: lotfi.benziane@ens.uvsq.fr

Contract/grant sponsor: This research was partially supported by the iCODE institute, research project of the Idex ParisSaclay; contract/grant number:

Copyright (C) 0000 John Wiley \& Sons, Ltd.

Prepared using rncauth.cls [Version: 2010/03/27 v2.00] 
solutions to the attitude stabilization problem rely on the quaternion parametrization as a global (but non-unique) representation evolving in the unit sphere $\mathbb{S}^{3}$ (see, for instance, $[2,4,5]$ ).

Roughly speaking, attitude control schemes have a proportional-derivative structure where the derivative action is in terms of the angular velocity. Designing attitude control schemes without angular velocity measurements is a more challenging problem that has been solved, for instance, in [12-19]. Removing the requirement of the angular velocity in the feedback allows to spare the use of gyroscopes which can be prone to failure and very expensive such as those used in satellites or sophisticated space telescopes. For instance, space telescope Hubble is equipped with six gyroscopes among which three are used as backups. In 2009, all six of Hubble's gyroscopes had to be replaced and one can imagine the cost generated. Therefore, it is conceivable to reduce costs and ensure continuity of a spacecraft mission despite the failure of the gyroscopes when velocityfree controllers are used. In most of existing velocity-free control schemes, the attitude is explicitly used in the feedback as a proportional action. As there is no sensor which physically measures the attitude of a rigid body, the aforementioned velocity-free controllers require some kind of attitude determination algorithm or attitude estimator relying on the available direction sensors. It is well known that static attitude determination algorithms are very sensitive to noise [20-22]. On the other hand, dynamic attitude estimators, relying on gyro and body-referenced vector measurements, such as Kalman filters and complementary filters are very efficient in the presence of noise [23-25]. Therefore, velocity-free attitude control schemes using body-referenced vector measurements in the feedback would indirectly require the angular velocity to efficiently recover the attitude. To overcome this problem, a velocity-free attitude control scheme, that incorporates explicitly vector measurements instead of the attitude itself, has been proposed for the first time in [26] leading to almost global asymptotic stability results*

A velocity-free attitude stabilization scheme relying solely on body-referenced vector measurements leading to almost global stability results is proposed in the present paper. The proposed solution, which extends preliminary work that appeared in [28], can be regarded as an improved version of [26]. The main differences are as follows: (a) the use of an auxiliary system in terms of body vector measurements, rather than an auxiliary system in terms of unit-quaternion; (b) An explicit design of an angular velocity observer-like system which is used in the design of the stabilizing feedback.

As a consequence, the set of unstable equilibria of the closed loop dynamics is reduced as compared to that of [26]. It is also shown that the proposed auxiliary error system does not make use of inertial fixed reference vectors as in [26], and that the introduction of gain matrices improves the controller performance. Moreover, it is shown that if at least two observed vectors are non-collinear, then there always exist positive control gains leading to almost global asymptotic stability results. Finally, in order to adjust properly the controller gains, a constrained non-linear optimal tuning method is used.

\footnotetext{
*Since it is impossible to achieve a global asymptotic attitude stabilization using continuous time-invariant state feedback [27], the strongest result that one can achieve in the rotational motion space is "Almost Global Asymptotic Stability" (AGAS). This notion is used in the sense that the desired equilibrium is asymptotically stable for all initial conditions belonging to an open and dense set in $S O(3)$.
} 


\section{NOTATIONS AND PROBLEM FORMULATION}

\subsection{Notations}

The attitude is represented by a rotation matrix $R$ or a unit-quaternion $Q=\left[q_{0}, q^{T}\right]^{T}$. Let $R \in$ $S O(3)=\left\{R \in \mathbb{R}^{3 \times 3} \mid R^{T} R=R R^{T}=I_{3 \times 3}, \operatorname{det}(R)=1\right\}$ and $Q \in \mathbb{S}^{3}=\left\{Q \in \mathbb{R}^{4} \mid Q^{T} Q=1\right\}$. The multiplication of two quaternion $P=\left(p_{0}, p^{T}\right)^{T}$ and $Q=\left(q_{0}, q^{T}\right)^{T}$ is denoted by “ $\odot$ " and defined as $P \odot Q=\left[\begin{array}{c}p_{0} q_{0}-p^{T} q \\ p_{0} q+q_{0} p+p \times q\end{array}\right]$. The Lie algebra of $S O(3)$ is denoted by $\mathfrak{s o}(3)$, i.e., the set of skew symmetric matrices $S$ generated via the Lie algebra isomorphism from $\mathbb{R}^{3} \rightarrow \mathfrak{s o}(3)$ which associates to $x=\left[x_{1}, x_{2}, x_{3}\right]^{T}$ the skew-symmetric matrix $S(x)$ given by

$$
S(x)=\left[\begin{array}{ccc}
0 & -x_{3} & x_{2} \\
x_{3} & 0 & -x_{1} \\
-x_{2} & x_{1} & 0
\end{array}\right] \text {. }
$$

Note that for every $x, y \in \mathbb{R}^{3}$, one has $S(x) y=x \times y$ where $\times$ stands for the vector cross product.

The mapping $\mathscr{R}: \mathbb{S}^{3} \rightarrow S O(3)$ given by Rodrigues' rotation formula [31]

$$
\mathscr{R}(Q)=I_{3 \times 3}+2 q_{0} S(q)+2 S(q)^{2}
$$

defines a double covering map of $S O(3)$ by $\mathbb{S}^{3}$, i.e., for every $R \in S O(3)$ the equation $\mathscr{R}(Q)=R$ admits exactly two solutions $Q_{R}$ and $-Q_{R}$. As a consequence, a vector field $f$ of $\mathbb{S}^{3}$ projects onto a vector field of $S O(3)$ if and only if, for every $Q \in \mathbb{S}^{3}, f(-Q)=-f(Q)$ (where the obvious identification between $T_{Q} \mathbb{S}^{3}$ the tangent space of $\mathbb{S}^{3}$ at $Q$ and $T_{-Q} \mathbb{S}^{3}$ the tangent space of $\mathbb{S}^{3}$ at $-Q$ was made), for more details see [29, 30].

In what follows and for simplicity, the notations below are used.

- If $m$ is a positive integer, $M_{m}(\mathbb{R})$ is used to denote the set of $m$ by $m$ matrices with real entries; $0_{3}, 0_{3 n}, 0$ and $I$ denote the 3 by 3 zero matrix, the $3 n$ by 1 zero vector, the 3 by 1 zero vector and the 3 by 3 identity matrix respectively;

- $\{\mathscr{B}\}$ and $\{\mathscr{I}\}$ denote an orthonormal body-attached frame with its origin at the center of mass of the rigid-body and the inertial reference frame on earth respectively.

For every $x, y \in \mathbb{R}^{3}$ and a given $R \in S O(3)$ one has the following [31]

$$
\begin{aligned}
S(x) y & =-S(y) x, S(x) x=0, \\
S(x) S(y) & =y x^{T}-x^{T} y I, S^{2}(x)=x x^{T}-x^{T} x I, \\
S(S(x) y) & =S(x) S(y)-S(y) S(x), S(R x)=R S(x) R^{T} .
\end{aligned}
$$

\subsection{Problem formulation}

The attitude of a rigid body is governed by

$$
\dot{R}(t)=R(t) S(\omega(t)),
$$


where $R \in S O(3)$. The equivalent kinematics evolving in $\mathbb{S}^{3}$ are given by

$$
\dot{Q}(t)=\left[\begin{array}{c}
\dot{q}_{0}(t) \\
\dot{q}(t)
\end{array}\right]=\left[\begin{array}{c}
-\frac{1}{2} q^{T}(t) \omega(t) \\
\frac{1}{2}\left(q_{0}(t) I+S(q(t))\right) \omega(t)
\end{array}\right],
$$

with $\omega(t)$ being the angular velocity of the rigid body expressed in $\{\mathscr{B}\}$ and $Q \in \mathbb{S}^{3}$ being the unit quaternion. Let $n \geq 2$ be an integer and let $b_{i}(Q(t))=b_{i}(t) \in \mathbb{R}^{3}(i=1, \ldots, n)$ be a measured vector expressed in $\{\mathscr{B}\}$. The relation between $b_{i}(t)$ and its corresponding inertial vector $r_{i} \in \mathbb{R}^{3}$ is given by

$$
b_{i}(t)=R^{T}(t) r_{i}
$$

Using (2) and (4), one can obtain the reduced attitude kinematics

$$
\dot{b}_{i}(t)=S\left(b_{i}(t)\right) \omega(t), i=1, \ldots, n
$$

The simplified rigid body rotational dynamics are governed by

$$
J \dot{\omega}(t)=-S(\omega(t)) J \omega(t)+\tau(t),
$$

where

- $J \in \mathbb{R}^{3 \times 3}$ is a symmetric positive definite constant inertia matrix about the center of mass of the rigid body expressed in $\{\mathscr{B}\}$;

- $\tau(t)$ is the external torque applied about the center of mass of the rigid body expressed in $\{\mathscr{B}\}$;

The problem addressed in this work is the design of an attitude stabilizing control $\tau(t)$ relying only on vector measurements $b_{i}(t)$, without using the angular velocity $\omega(t)$ in the feedback.

\subsection{Assumptions}

The following assumptions are made for the rest of the paper.

A1. Assume that only the $n$ vector-valued functions of time $b_{i}(t)$ are measured and no similar assumption are made on angular velocity vector $\omega(t)$. Moreover, note that the $b_{i}$ 's actually depend on the rotation $R$ and one could also write them as $b_{i}(R(t))$ or $b_{i}(Q(t))$. In the sequel, notations $b_{i}(t)$ or $b_{i}(Q(t))$ will be used equivalently.

A2. At least two vectors $r_{1}, r_{2}$ are non-collinear. As a consequence, $b_{1}(t)$ and $b_{2}(t)$ are linearly independent for all non negative times.

$A 3$. The desired rigid body attitude is defined by the constant rotation matrix $R_{d}$. It relates an inertial vector $r_{i}$ to its corresponding vector in the desired frame, i.e., $b_{i}^{d}=R_{d}^{T} r_{i}$, with $\dot{b}_{i}^{d}=0$. An equivalent constant desired unit-quaternion $Q_{d}$ is defined as $R_{d}=\mathscr{R}\left(Q_{d}\right)$. 


\section{HANDLING THE LACK OF ANGULAR VELOCITY AND DESIGN OF THE ATTITUDE CONTROLLER}

\subsection{Angular velocity observer-like system}

As well known, the reduced attitude kinematic is defined by (5). Let $\Gamma=\operatorname{diag}\left(\Lambda_{1}, \ldots, \Lambda_{n}\right)$, where $\Lambda_{i}$ be a symmetric positive definite $3 \times 3$ matrix, for $1 \leq i \leq n$. Let $M(t)=\sum_{i=1}^{n} S\left(b_{i}(t)\right)^{T} \Lambda_{i} S\left(b_{i}(t)\right)$, which is a symmetric positive definite matrix, thanks to Assumption $A 2$.

Multiplying (5) by $S\left(b_{i}(t)\right) \Lambda_{i}$ for $1 \leq i \leq n$ and summing, yields

$$
\sum_{i=1}^{n} S\left(b_{i}(t)\right) \Lambda_{i} \dot{b}_{i}(t)=-M(t) \omega(t)
$$

From (7), the actual angular velocity $\omega(t)$ is given by

$$
\omega(t)=-M^{-1}(t) \sum_{i=1}^{n} S\left(b_{i}(t)\right) \Lambda_{i} \dot{b}_{i}(t)
$$

Since $\dot{b}_{i}(t)$ is not a measured quantity, the following angular velocity observer-like signal is proposed

$$
\hat{\omega}(t)=-M^{-1}(t) \sum_{i=1}^{n} S\left(b_{i}(t)\right) \Lambda_{i} \dot{\hat{b}}_{i}(t)
$$

where the vector $\dot{\hat{b}}_{i}(t)$ can be viewed as an estimate of the vector $\dot{b}_{i}(t)$ using the following linear first-order filter on $b_{i}(i=1, \ldots, n)$

$$
\dot{\hat{b}}_{i}(t)=A_{i}\left(b_{i}(t)-\hat{b}_{i}(t)\right)
$$

where the constant matrices $A_{i} \in \mathbb{R}^{3 \times 3}$ are chosen as $A_{i}=P_{i}\left(\Lambda_{i}\right)$, for $1 \leq i \leq n$, with $P_{i}$ being a positive polynomial of degree two on $\mathbb{R}_{+}^{*}$, and $\mathbb{R}_{+}^{*}$ denotes the set of strictly positive real numbers. As a trivial consequence, $A_{i}, 1 \leq i \leq n$, is symmetric positive definite and commutes with $\Lambda_{i}$. Let $A \triangleq \operatorname{diag}\left(A_{1}, \cdots, A_{n}\right), \Gamma_{d} \triangleq \operatorname{diag}\left(R_{d} \Lambda_{1} R_{d}^{T}, \cdots, R_{d} \Lambda_{n} R_{d}^{T}\right)$ and $A_{d} \triangleq \operatorname{diag}\left(R_{d} A_{1} R_{d}^{T}, \cdots, R_{d} A_{n} R_{d}^{T}\right)$. Then $\Gamma_{d}$ and $A_{d}$ commute.

Defining the error signal $\tilde{b}_{i}(t)=b_{i}(t)-\hat{b}_{i}(t)$, and using (10) and (5) one has $\dot{\tilde{b}}_{i}(t)=-A_{i} \tilde{b}_{i}(t)+$ $S\left(b_{i}(t)\right) \omega$, which can be rewritten using the state vector defined by $\zeta(t):=\left[\tilde{b}_{1}^{T}(t), \cdots, \tilde{b}_{n}^{T}(t)\right]^{T}$, as

$$
\dot{\zeta}(t)=-A \zeta(t)+B(t) \omega(t)
$$

where $B(t)=\left[\begin{array}{lll}S\left(b_{1}(t)\right)^{T} & \cdots & S\left(b_{n}(t)\right)^{T}\end{array}\right]^{T}$. Finally, the angular velocity observer-like signal can be written as

$$
\hat{\omega}(t)=M^{-1}(t) B^{T}(t) \Gamma A \zeta(t) .
$$




\subsection{Controller Design}

The orientation error is defined by

$$
\bar{R}(t)=R(t) R_{d}^{T}
$$

where $R(t)$ is a rotation matrix and $R_{d}$ is a constant desired rotation matrix. From (2) and (13) one can obtain the attitude error dynamics as follows

$$
\dot{\bar{R}}(t)=\bar{R}(t) S\left(R_{d} \omega(t)\right)
$$

The corresponding quaternion error $\bar{Q}(t)=Q(t) \odot Q_{d}^{-1}(t) \equiv\left[\bar{q}_{0}(t), \bar{q}(t)^{T}\right]^{T}$ whose dynamics are governed by

$$
\left[\begin{array}{c}
\dot{\bar{q}}_{0}(t) \\
\dot{\bar{q}}(t)
\end{array}\right]=\left[\begin{array}{c}
-\frac{1}{2} \bar{q}^{T}(t) R_{d} \omega(t) \\
\frac{1}{2}\left(\bar{q}_{0}(t) I+S(\bar{q}(t))\right) R_{d} \omega(t)
\end{array}\right] .
$$

The reduced orientation error is given by $\bar{b}_{i}(t)=b_{i}(t)-b_{i}^{d}$. Therefore, one can get

$$
\bar{b}_{i}(t)=R_{d}^{T}\left(\bar{R}(t)^{T}-I\right) r_{i}
$$

where $1 \leq i \leq n$ which can be rewritten using (1) as

$$
\bar{b}_{i}(t)=\bar{b}_{i}(\bar{Q}(t))=-2 R_{d}^{T}\left(\bar{q}_{0}(t) I-S(\bar{q}(t))\right) S(\bar{q}(t)) r_{i}
$$

The following control law is proposed

$$
\tau(t)=z_{\rho}(t)-M \hat{\omega}(t)
$$

where the term $z_{\rho}(\cdot)$ was introduced in [26] and is given by

$$
z_{\rho}(t)=\sum_{i=1}^{n} \rho_{i} S\left(b_{i}^{d}\right) b_{i}
$$

where the coefficients $\rho_{i}$ 's are positive constants. Define

$$
W_{\rho}=-\sum_{i=1}^{n} \rho_{i} S^{2}\left(r_{i}\right)
$$

which is a symmetric positive definite matrix, see Lemma 2 in [26]. It has been shown in Lemma 1 of [26] that $z_{\rho}(\cdot)$ can be rewritten as

$$
z_{\rho}(t)=-2 R_{d}^{T}\left(\bar{q}_{0}(t) I-S(\bar{q}(t))\right) W_{\rho} \bar{q}(t) .
$$

Consequently, $\tau(\cdot)$ can be rewritten as

$$
\tau(t)=-2 R_{d}^{T}\left(\bar{q}_{0}(t) I-S(\bar{q}(t))\right) W_{\rho} \bar{q}(t)-M \hat{\omega}(t) .
$$


Using (11), (15), (6) and (22), one can obtain the following closed loop dynamics

$$
\left\{\begin{array}{l}
\dot{\zeta}=-A \zeta+B(\bar{Q}) \omega \\
\dot{\bar{q}}_{0}=-\frac{1}{2} \bar{q}^{T} R_{d} \omega, \\
\dot{\bar{q}}=\frac{1}{2}\left(\bar{q}_{0} I+S(\bar{q})\right) R_{d} \omega, \\
J \dot{\omega}=-S(\omega) J \omega-2 R_{d}^{T}\left(\bar{q}_{0} I-S(\bar{q})\right) W_{\rho} \bar{q}-M \hat{\omega} .
\end{array}\right.
$$

One can make further simplifications by changing variables as follows

$$
\zeta \rightarrow \xi=\left[\left(R_{d} \tilde{b}_{1}(Q(t))\right)^{T}, \cdots,\left(R_{d} \tilde{b}_{n}(Q(t))\right)^{T}\right]^{T}, \quad \omega \rightarrow \bar{\omega}=R_{d} \omega
$$

By setting

$$
J_{d}:=R_{d} J R_{d}^{T}, B_{d}:=\left[\begin{array}{lll}
S\left(R_{d} b_{1}\right)^{T} & \cdots & S\left(R_{d} b_{n}\right)^{T}
\end{array}\right]^{T},
$$

one can end up with the following autonomous dynamics

$$
\left\{\begin{array}{l}
\dot{\xi}=-A_{d} \xi+B_{d} \bar{\omega}, \\
\dot{\bar{q}}_{0}=-\frac{1}{2} \bar{q}^{T} \bar{\omega}, \\
\dot{\bar{q}}=\frac{1}{2}\left(\bar{q}_{0} I+S(\bar{q})\right) \bar{\omega}, \\
J_{d} \dot{\bar{\omega}}=-S(\bar{\omega}) J_{d} \bar{\omega}-2\left(\bar{q}_{0} I-S(\bar{q})\right) W_{\rho} \bar{q}-B_{d}^{T} \Gamma_{d} A_{d} \xi .
\end{array}\right.
$$

Note that $J_{d}$ is a real symmetric positive definite matrix. If one defines the state $\chi:=(\xi, \bar{Q}, \bar{\omega})$ and the state space $\Upsilon:=\mathbb{R}^{3 n} \times \mathbb{S}^{3} \times \mathbb{R}^{3}$, one can rewrite (24) as $\dot{\chi}=F(\chi)$ where $F$ gathers the right-hand side of (24) and defines a smooth vector field on $\Upsilon$. Moreover, note that $\bar{Q}$ and $-\bar{Q}$ represents the same physical rotation, implying that (24) projects on $S O(3)$ as an autonomous differential equation.

Lemma 1. With the notations above, the set of gains $\rho=\left(\rho_{1}, \cdots, \rho_{n}\right)$ leading to simple eigenvalues of the matrix $W_{\rho}$ is dense and open in $\left(\mathbb{R}_{+}^{*}\right)^{n}$.

Proof

For $\rho \in\left(\mathbb{R}_{+}^{*}\right)^{n}$, let $P_{\rho}(\cdot)$ be the characteristic polynomial of $W_{\rho}$ and $\Delta(\rho)$ its discriminant [32]. Recall that $\Delta(\rho)=0$ if and only if $P_{\rho}(\cdot)$ admits a multiple root. Since $W_{\rho}$ is a 3 by 3 real symmetric positive definite matrix for every $\rho \in\left(\mathbb{R}_{+}^{*}\right)^{n}, \Delta(\rho)$ is actually a homogeneous polynomial of degree six in $\rho$ (for more details about the evaluation of the discriminant see page 102 of [33]). Thus the locus $\Delta(\rho)=0$ defines an algebraic variety of co-dimension one in $\left(\mathbb{R}_{+}^{*}\right)^{n}$ (see, for instance, [34]) and, on its complementary set $\mathscr{S}$ in $\left(\mathbb{R}_{+}^{*}\right)^{n}, W_{\rho}$ has simple eigenvalues, which means that the set of gains $\rho$ leading to simple eigenvalues of $W_{\rho}$ is dense and open in $\left(\mathbb{R}_{+}^{*}\right)^{n}$.

This genericity result serves a justification to the following working hypothesis, which will hold for the rest of the paper.

(GEN) $W_{\rho}$ has simple eigenvalues. 


\section{STABILITY ANALYSIS OF THE PROPOSED CONTROLLER}

As often, it turns out that it is simpler for the stability analysis to use unit quaternion for the attitude representation instead of elements of $S O(3)$, even-though a result formulated in terms of orthogonal matrices is interesting. This is why the stability analysis is completed using unit quaternion and a theorem (Theorem 1) is obtained, after it will be straightforward to state the main result in terms of elements of $S O(3)$ by simply projecting Theorem 1 using Rodriguez formula (1).

Lemma 2. Under the hypothesis (GEN), the solutions of equation $z_{\rho}=0$ where $z_{\rho}$ is defined by (21) are the following: (a) the two points $\pm(1,0)$; the six points $\pm\left(0, v_{i}\right), 1 \leq i \leq 3$, with $\left(v_{1}, v_{2}, v_{3}\right)$ being an orthonormal basis diagonalizing $W_{\rho}$.

Proof

Let $\left(q_{0}, q\right) \in \mathbb{S}^{3}$ such that $z_{\rho}=0$, i.e.,

$$
\left(q_{0} I-S(q)\right) W_{\rho} q=0
$$

If $q_{0} \neq 0$, it is immediate to see that $q_{0} I-S(q)$ is invertible and thus $q=0$, implying that $q_{0}= \pm 1$. If $q_{0}=0$, then $S(q) W_{\rho} q=0$. According to the properties of $S(q)$ with $q \in \mathbb{S}^{2}$ and using (GEN), it is clear that $q$ is an eigenvector of $W_{\rho}$ with unit length.

Consider the following positive definite differentiable function $V: \Upsilon \rightarrow \mathbb{R}^{+}$

$$
V=\xi^{T} \Gamma_{d} A_{d} \xi+4 \bar{q}^{T} W_{\rho} \bar{q}+\bar{\omega}^{T} J_{d} \bar{\omega}
$$

which is radially unbounded over $\Upsilon$ since $W_{\rho}$ and $J_{d}$ are positive definite. Moreover, since $\Gamma_{d}$ and $A_{d}$ commute, the gain matrix $\Gamma_{d} A_{d}$ is symmetric block diagonal positive definite.

Theorem 1. Consider the system (3)-(6), under the assumptions in subsection (2.3) and the control law (22) with the auxiliary system given by (11). Then, under hypothesis (GEN), the following hold

(1) There are eight equilibrium points, given by

$$
\begin{aligned}
\Omega_{1}^{+} & =\left(\mathbf{0}_{3 n},\left[\begin{array}{l}
1 \\
0
\end{array}\right], 0\right), \Omega_{1}^{-}=\left(\mathbf{0}_{3 n},\left[\begin{array}{c}
-1 \\
0
\end{array}\right], 0\right), \\
\Omega_{i+1}^{+} & =\left(\mathbf{0}_{3 n},\left[\begin{array}{c}
0 \\
v_{i}
\end{array}\right], 0\right), \Omega_{i+1}^{-}=\left(\mathbf{0}_{3 n},\left[\begin{array}{c}
0 \\
-v_{i}
\end{array}\right], 0\right),
\end{aligned}
$$

with $i=1,2,3$ and $\left(v_{1}, v_{2}, v_{3}\right)$ is an orthonormal basis diagonalizing $W_{\rho}$.

(2) All trajectories of (3)-(6) converge to one of the equilibrium points defined in Item (1).

(3) Set $c:=4 \lambda_{\min }\left(W_{\rho}\right)$, where $\lambda_{\min }\left(W_{\rho}\right)$ is the smallest eigenvalue of $W_{\rho}$, then the equilibrium point $\Omega_{1}^{+}$is locally asymptotically stable with a domain of attraction containing the set

$$
V_{c}^{+}:=\left\{\chi \in \Upsilon \mid V(\chi)<c: \text { and } \bar{q}_{0}>0\right\},
$$


and the equilibrium point $\Omega_{1}^{-}$is locally asymptotically stable with a domain of attraction containing the set

$$
V_{c}^{-}:=\left\{\chi \in \Upsilon \mid V(\chi)<c \text { and } \bar{q}_{0}<0\right\}
$$

(4) The other equilibrium points $\Omega_{2,3,4}^{ \pm}$are hyperbolic and not stable (i.e., the eigenvalues of each of the corresponding linear systems have non zero real part and at least one of them has positive real part). This implies that System (3)-(6) is almost globally asymptotically stable with respect to the two equilibrium points $\Omega_{1}^{ \pm}$in the following sense: there exists an open and dense subset $\Upsilon_{0} \subset \Upsilon$ such that, for every initial condition $\chi_{0} \in \Upsilon_{0}$, the corresponding trajectory converges asymptotically to either $\Omega_{1}^{+}$or $\Omega_{1}^{-}$.

Proof

Regarding Item (1), one must solve the equation $f(\chi)=0$, where $f$ is the nonlinear function describing (24). Two cases can be considered. Assume first that $\bar{q}_{0} \neq 0$. Both matrices $\bar{q}_{0} I+S(\bar{q})$ and $\bar{q}_{0} I-S(\bar{q})$ are non singular. Therefore, from the third equation of (24) $\bar{\omega}=0$ and thus $\xi=\mathbf{0}_{3 n}$ from the first equation of (24). The fourth equation of (24) reduces to $z_{\rho}=0$ and one concludes that $\bar{q}=0$ and $\bar{q}_{0}= \pm 1$ leading to two equilibrium points : $\Omega_{1}^{+}=\left(\mathbf{0}_{3 n},\left[\begin{array}{l}1 \\ 0\end{array}\right], 0\right)$ and $\Omega_{1}^{-}=\left(\mathbf{0}_{3 n},\left[\begin{array}{c}-1 \\ 0\end{array}\right], 0\right)$.

Now, assume that $\bar{q}_{0}=0$. Then $\|\bar{q}\|=1$ and according to the third equation of (24), it is clear that $\bar{\omega}$ is parallel to $\bar{q}$, let say $\bar{\omega}=\mu \bar{q}$ and then $\mu$ must be equal to zero according to the second equation of (24), implying that $\bar{\omega}=0$. As in the previous case, one deduces that $\xi=\mathbf{0}_{3 n}$. From the fourth equation of (24) one can conclude that $\bar{q}$ and $W_{\rho} \bar{q}$ are parallel, leading to the six points $\Omega_{2,3,4}^{ \pm}$.

Regarding Item (2). Using the facts that

$$
\bar{\omega}^{T} S(\bar{\omega})=0, \bar{q}^{T} W_{\rho}\left(\bar{q}_{0} I+S(\bar{q})\right) \bar{\omega}=\bar{\omega}^{T}\left(\bar{q}_{0} I-S(\bar{q})\right) W_{\rho} \bar{q}, \bar{\omega}^{T} B_{d}^{T} \Gamma_{d} \xi=\xi^{T} \Gamma_{d} B_{d} \bar{\omega},
$$

the time derivative of (25) in view of (24) yields

$$
\dot{V}=-\xi^{T} \Lambda \xi \leq 0,
$$

with $\Lambda=A_{d}^{T} \Gamma_{d} A_{d}+\Gamma_{d} A_{d}^{2}=2 \Gamma_{d} A_{d}^{2}$ symmetric positive definite. Therefore, all trajectories of (24) are defined for all times and bounded.

Since (24) is autonomous and $V$ is radially unbounded, one can use LaSalle's invariance theorem. Therefore every trajectory converges to a trajectory $\gamma$ along which $\dot{V} \equiv 0$. Then $\xi$ must be identically equal to zero, implying at once that $B_{d} \bar{\omega} \equiv 0$ as well. The latter assertion yields that $\bar{\omega}$ must be collinear to all the $b_{i}$ 's, which can be true only if $\bar{\omega} \equiv 0$ since there are at least two non-collinear vectors $b_{i}$. From the fourth equation of (24) one can conclude that $z_{\rho}=0$ leading to the conclusion by Lemma 2 .

Next Item (3) is addressed. The proof is provided only for $\Omega_{1}^{+}$since the other case is entirely similar. Take an initial condition $\bar{\chi}$ in $V_{c}^{+}$. Since $V$ is non increasing, $V(\chi)<c$ for all times and, for every $t \geq 0, \bar{q}(t)^{T} W_{\rho} \bar{q}(t) \leq \lambda_{\min }\left(W_{\rho}\right)$. This implies that $\|\bar{q}(t)\|<1$ for every $t \geq 0$ and thus $\bar{q}_{0}(t) \neq 0$ for every $t \geq 0$. One can deduce that $\bar{q}_{0}(t)$ keeps the same sign namely that $\bar{q}_{0}(0)$, which is positive. Since the trajectory converges to one of the eight equilibrium points, it must be $\Omega_{1}^{+}$since this is the only one contained in $V_{c}^{+}$.

Finally, the proof for Item (4) is deferred to Appendix A. 
Remark 1. Denote by $\Psi \subset \Upsilon$ the set composed of the union of stable manifolds of the unstable equilibria $\Omega_{j}^{ \pm}, j=2,3,4$. Therefore, for every initial condition $\chi_{0} \in \Psi$, the corresponding trajectory converges to one of the unstable equilibrium points $\Omega_{j}^{ \pm}, j=2,3,4$.

\section{CONTROL GAINS TUNING AND SIMULATION RESULTS}

This section provides an optimization procedure for the control gains tuning. Let $\chi=$ $\left(\left[\begin{array}{l}\tilde{b}_{1} \\ \tilde{b}_{2}\end{array}\right], Q, \omega\right)$, where only two non-collinear vectors $b_{1}$ and $b_{2}$ were considered. For simplicity and without loss of generality $R_{d}=I$, which means that $\bar{q}=q, \bar{\omega}=\omega$ and $b_{i}^{d}=r_{i}$. The matrices $\Lambda_{1}, \Lambda_{2}$ are chosen diagonal such as $\Lambda_{i}=\operatorname{diag}\left(\gamma_{i 1}, \gamma_{i 2}, \gamma_{i 3}\right)$ where $i=1,2$, therefore the matrices $A_{1}$ and $A_{2}$ will be $A_{i}=a_{i 0} I+a_{i 1} \Lambda_{i}+a_{i 2} \Lambda_{i}^{2}$ where $i=1,2$. The inertial reference vectors are taken as $r_{1}=[0,0,1]^{T}$ and $r_{2}=[0.4348,0.0008,0.9005]$, and the inertia matrix is selected from [35]

$$
J=\left[\begin{array}{ccc}
10 & 1.2 & 0.5 \\
1.2 & 19 & 1.5 \\
0.5 & 1.5 & 25
\end{array}\right]\left(K g . \mathrm{m}^{2}\right)
$$

and the simulation sampling time is $0.01 \mathrm{~s}$ with ode5 solver.

\subsection{Parameters Tuning}

Consider the closed-loop dynamics is given by (24), and consider the case where only two noncollinear inertial vectors $r_{1}, r_{2}$ are used. Consider now an objective function $g(\kappa)$, where $\kappa$ is the vector of all parameters to be tuned. The goal is to find $\min _{\kappa}(g(\kappa))$ with the following constraint $l(\kappa(\cdot)) \leq \kappa(\cdot) \leq u(\kappa(\cdot))$, where $\kappa=\left[\begin{array}{llllll}\rho_{1} & \rho_{2} & a_{1(j-1)} & a_{2(j-1)} & \gamma_{1 j} & \gamma_{2 j}\end{array}\right]^{T},(j=$ $\left.1, \ldots, 3, \kappa \in\left(\mathbb{R}_{+}^{*}\right)^{14}\right)$ is the vector of parameters, $l(\kappa(\cdot))$ and $u(\kappa(\cdot))$ are the lower and upper bounds corresponding to each parameter and $\kappa(\cdot)$ is an element of $\kappa$.

Usually, existing optimization algorithms find a local optimum and there effectiveness depends on the lower and upper limits. These last values can be determined based on the dominant poles of the linearized system around the stable equilibrium point.

The linearization of (24) at $\Omega_{1}^{+}=\left(\mathbf{0}_{6},\left[\begin{array}{l}1 \\ \mathbf{0}\end{array}\right], \mathbf{0}\right)$ can be written as follows

$$
\left\{\begin{array}{l}
\dot{z}_{\xi}=-A z_{\xi}+G z_{\omega} \\
\dot{z}_{q}=\frac{1}{2} z_{\omega} \\
J \dot{z}_{\omega}=-G^{T} \Gamma A z_{\xi}-2 W_{\rho} z_{q}
\end{array}\right.
$$

where $G=\left[\begin{array}{cc}G_{1}^{T} & G_{2}^{T}\end{array}\right]^{T}$ with $G_{i}=S\left(\left(I-2 q_{0} S(q)+2 S^{2}(q)\right) r_{i}\right)_{\mid \Omega_{1}^{+}}=S\left(r_{i}\right), \Gamma$ and $A$ are defined in subsection 3.1 and $W_{\rho}$ is defined in (20). Setting $Z=\left(z_{\xi}^{T}, z_{q}^{T}, z_{\omega}^{T}\right)^{T}$ where $z_{\xi} \in \mathbb{R}^{6}, z_{q} \in \mathbb{R}^{3}$ and $z_{\omega} \in \mathbb{R}^{3}$ are the linearized vectors of $\xi, q, \omega$, respectively. Then system (29) can be rewritten as 
$\dot{Z}=\mathscr{B} Z$, where

$$
\mathscr{B}=\left[\begin{array}{ccc}
-A & 0_{6 \times 3} & G \\
0_{3 \times 6} & 0_{3} & I / 2 \\
-J^{-1} G^{T} \Gamma A & -2 J^{-1} W_{\rho} & 0_{3}
\end{array}\right] .
$$

Note that the fact that $\dot{z}_{q_{0}}=0$ was used. The linearization of the closed loop dynamics is used to determine the upper and the lower limits $u(\kappa(\cdot)), l(\kappa(\cdot))$ respectively. An arbitrary initial condition $Q(0)=[0.7212,0.3999,-0.3999,0.3999]^{T}$ is selected and for an arbitrary chosen fixed $\kappa(m), m=3, \ldots, 14$ gains values, one can start by varying $\kappa(1)$ and $\kappa(2)$. After inspecting the zero-pole map, one can determine the upper and lower bounds for $\kappa(1)$ and $\kappa(2)$ gains based on the location of the dominant poles, if they exist. The same reasoning gives the values in Table I.

Since there exist many possibilities to select the objective function. Different objective functions derived from three well known performance indices (see section 5.7 of [36]) were tested. The first is Integral of Absolute Error (IAE), the second is Integral of Time-weighted Absolute Error (ITAE) and the last is Integral of Square Error (ISE), with the possibility to minimize energy and attitude error in the same time by choosing $\sigma \in\left[\begin{array}{ll}0 & 1\end{array}\right]$. The first conclusion after several simulations is that the most appropriate objective function for the considered application is the ISE function $g_{i s e}(\kappa)=\int_{0}^{\infty}\left(\|\bar{q}\|^{2}+\sigma\|\tau\|^{2}\right) d t$ with $\sigma=0.1$. Initial gains vector are chosen arbitrary as $\kappa_{0}=$ $[0.599,0.586,0.754,0.368,0.015,5.668,1.963,0.099,2.155,5.561,0.021,5.979,5.464,5.954]$.

To get an idea about the effectiveness of the optimization methods, two methods have been compared. The first one uses Matlab fmincon function and the second method is based on the use of the same function with variation of initial conditions of the parameters in a procedure called global search (see, for instance [37]) because the locality of the solution essentially depends on the initial conditions. The global search method has been used and the final value $\kappa_{\text {final }}$ with criterion ISE is presented in Table II. The corresponding gain matrices are given in Table III. Note that the eigenvalues of the obtained matrix $W_{\rho}$ are simple, despite the fact that it is not included as a constraint in the tuning gains method. Also, during all conducted simulations using two noncollinear reference vectors no selected set of gains led to multiple eigenvalues of $W_{\rho}$.

\subsection{Simulation results}

The impact of the tuned gains on the behavior of the controller are shown in this subsection and the effectiveness of the proposed controller is illustrated via simulations. Depending on the attitude initial condition and measurement noise, four cases were selected.

- In the first case, the initial attitude is $Q(0)=[0.7212,0.3999,-0.3999,0.3999]^{T}$ without measurement noise.

- In the second case, the initial attitude is $Q(0)=[-0.7212,0.3999,-0.3999,0.3999]^{T}$ without measurement noise.

- The third case is chosen to be an unstable equilibrium point $Q(0)=[0,0,-1,0]^{T}$ without measurement noise.

- The fourth case uses the same initial condition as the first case, but a White Centered Gaussian noise with standard deviation of 0.01 (normalized) is added to the three elements of vector measurements $b_{1}$ and $b_{2}$. Note that $b_{1}$ and $b_{2}$ are not renormalized after the noise has been added to them. 
In all cases the initial angular velocity is chosen to be $\omega(0)=[0.005,0.006,0.004]^{T}(\mathrm{rad} / \mathrm{s})$.

Remark 2. Special care should be taken when dealing with noisy measurements. In the forth case the gain parameters are chosen differently, the gains $a_{1(j-1)}(j=1,2,3), a_{i 1}(i=$ $1,2)$ and $a_{i 2}(i=1,2)$ remain unchanged (see Table II), the selected other gains are $\Lambda_{1}=\operatorname{diag}([25.7484,19.4165,30.6847]), \Lambda_{2}=\operatorname{diag}([30.4728,15,10.8129])$ which gives $A_{1}=$ $\operatorname{diag}([3.6,2.3966,4.7274]), A_{1}=\operatorname{diag}([3.4217,1.2048,0.8271])$ and $\rho_{1}=6.0339, \rho_{2}=4.3266$. This choice is justified by the fact that matrices $A_{1}$ and $A_{2}$ represent the frequency cutoff of the auxiliary filters.

The evolution of the unit-quaternion trajectories, angular velocity and applied torques with respect to time for the proposed controller in case 1 are presented in Figure 1, where the trajectories converge asymptotically to the equilibrium point $\Omega_{1}^{+}$. Figure 2 illustrates simulation results in case 2 , where it is clear that the proposed controller avoids the unwinding phenomenon. In this case the trajectories converge asymptotically to the equilibrium point $\Omega_{1}^{-}$. Note that, in the third case, even if the initial condition is a theoretical unstable equilibrium point, by simulation, numerical errors push the trajectories away from this point as depicted in Figure 3. Figure 4 show that the presence of measurement noise does not affect the convergence of quaternion trajectories to the stable equilibrium point.
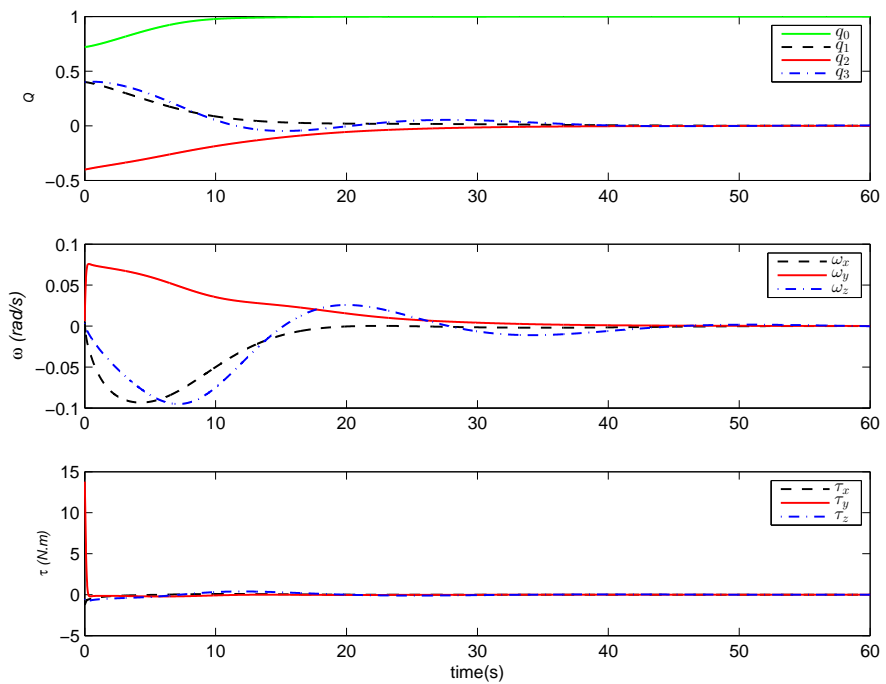

Figure 1. Simulation results for case 01

\section{CONCLUSIONS}

An attitude stabilization controller has been proposed, in which neither the angular velocity nor the attitude are used in the feedback. This controller could be of great help (as a main or a backup) controller in applications where prone-to-failure and expensive gyroscopes are used. Most existing velocity-free attitude stabilization schemes use directly the attitude in the feedback. However, an efficient recovery of the attitude requires the use of the angular velocity together with inertial vector measurements. The proposed velocity-free control schemes does not require the reconstruction of 

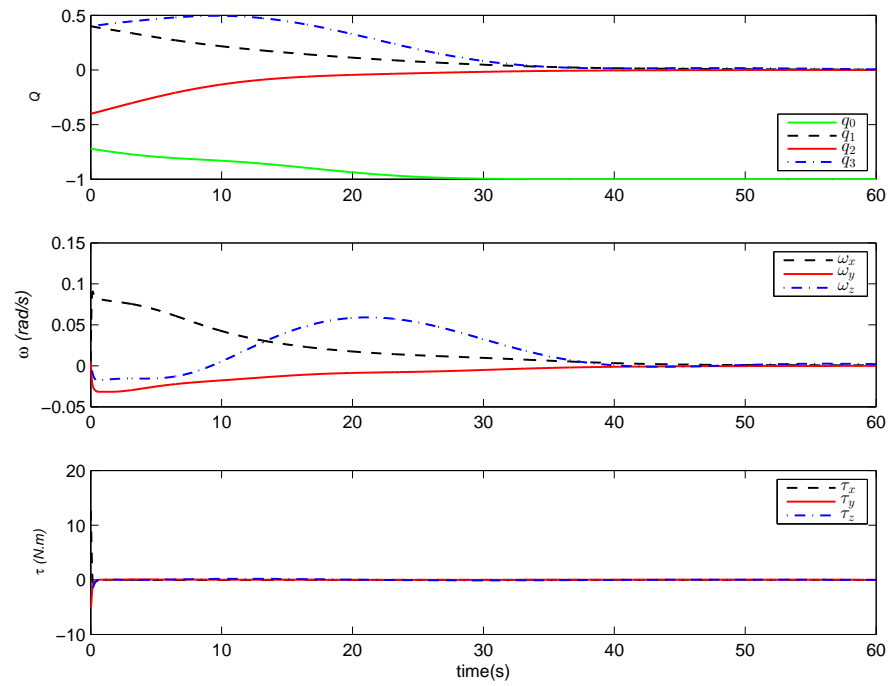

Figure 2. Simulation results for case 02
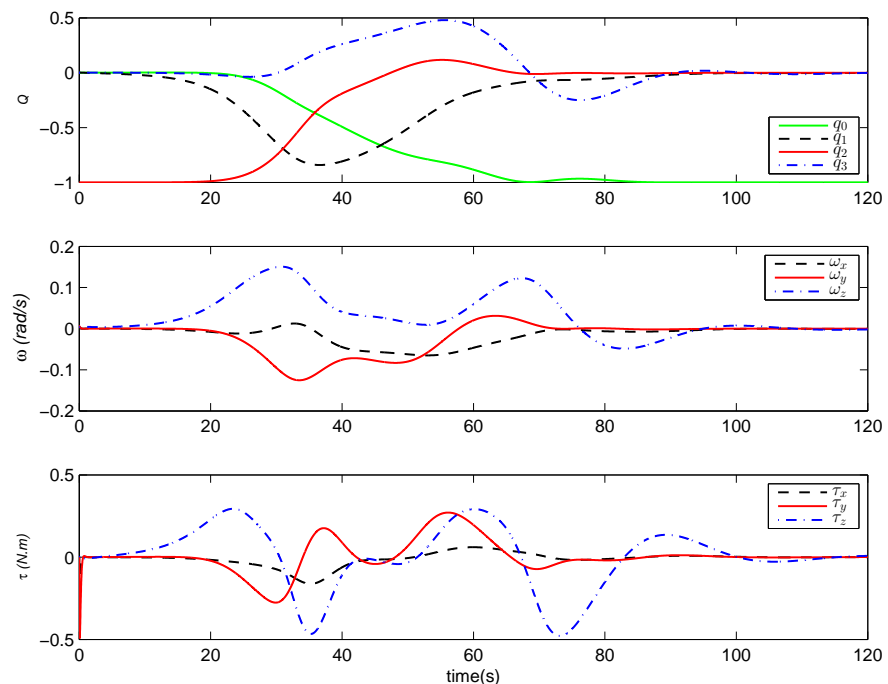

Figure 3. Simulation results for case 03

the attitude. It mainly uses an auxiliary system that can be considered as an observer-like for the angular velocity relying only on inertial vector measurements. The proposed controller doesn't use the inertial reference vectors, reduces the set of unstable equilibria of the closed loop dynamics with respect to previously proposed controllers, provides an almost global asymptotic stability of the desirable equilibrium point and avoids the "unwinding phenomenon". In addition, it was shown that if at least two observed vectors are non collinear, then there always exist positive control gains leading to almost global asymptotic stability results. A nonlinear optimal tuning method has been used to adjust properly the controller gains. 

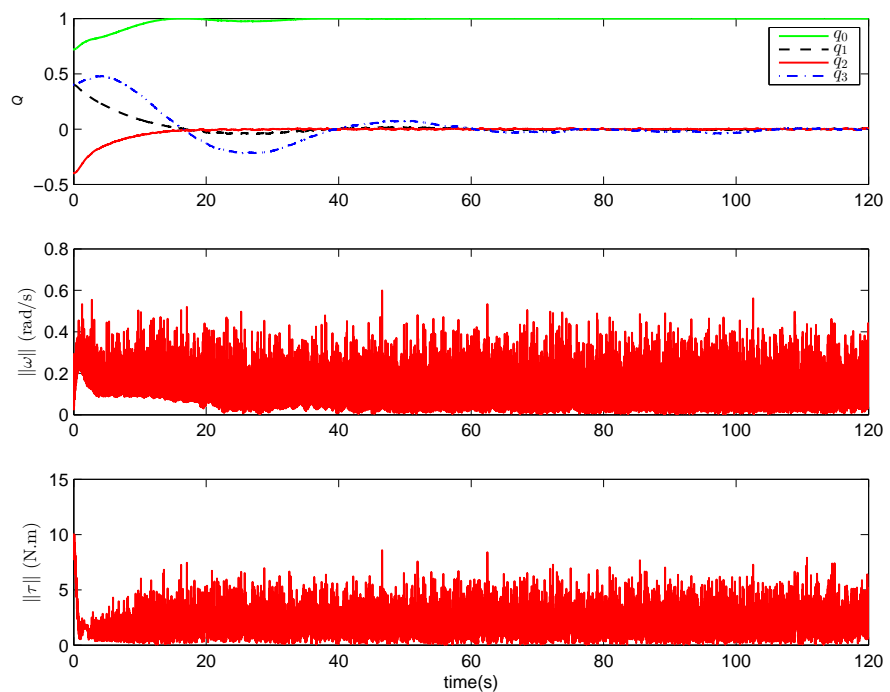

Figure 4. Simulation results for case 04

\section{APPENDIX A. PROOF OF ITEM 4 OF THEOREM 1}

Proof

First of all notice that the equilibrium points $\Omega_{i}^{ \pm}, i=2,3,4$, cannot be locally asymptotically stable. Indeed let $\Omega$ be one of these points and $U$ any open neighborhood of $\Omega$ in $\Upsilon$. Define

$$
V_{\Omega}^{-}:=\{\chi \in \Upsilon \mid V(\chi)<V(\Omega)\}
$$

and set $U^{-}:=\left(V_{\Omega}^{-} \cap U\right)$. The set $U^{-}$is obviously non empty since it contains points of the type $\lambda \Omega$ with $|\lambda|<1$ close enough to 1 . Moreover, for every $\chi \in U^{-}$, the trajectory of (24) does not converge to $\Omega$ since $V$ is non-increasing.

Next it is shown that the linearization of (24) at $\Omega$ is hyperbolic and admits an eigenvalue with positive real part. If $\bar{q}_{0}=0$ then $\bar{q}=\sigma v_{\rho}$, where $\sigma= \pm 1$ and $v_{\rho}$ is an eigenvector of $W_{\rho}$. Let one use the following change of variable (cf. [11, 24, 38])

$$
X=\left[\begin{array}{c}
x_{0} \\
x
\end{array}\right]=\left[\begin{array}{c}
0 \\
-\sigma v_{\rho}
\end{array}\right] \odot\left[\begin{array}{c}
\bar{q}_{0} \\
\bar{q}
\end{array}\right]=\sigma\left[\begin{array}{c}
v_{\rho}^{T} \bar{q} \\
-\bar{q}_{0} v_{\rho}-S\left(v_{\rho}\right) \bar{q}
\end{array}\right]
$$

From (30) one can have

$$
\left[\begin{array}{c}
\bar{q}_{0} \\
\bar{q}
\end{array}\right]=\left[\begin{array}{c}
0 \\
\sigma v_{\rho}
\end{array}\right] \odot\left[\begin{array}{c}
x_{0} \\
x
\end{array}\right]=\sigma\left[\begin{array}{c}
-v_{\rho}^{T} x \\
x_{0} v_{\rho}+S\left(v_{\rho}\right) x
\end{array}\right] .
$$

In the forth equation of (24), the term $\Xi=-\left(\bar{q}_{0} I-S(\bar{q})\right) W_{\rho} \bar{q}$ can be rewritten function of the new variable. First using the fact that $\sigma^{2}=1, W_{\rho} v_{\rho}=\lambda_{\rho} v_{\rho}$ and $S\left(S\left(v_{\rho}\right) x\right)=S\left(v_{\rho}\right) S(x)-S(x) S\left(v_{\rho}\right)$, 
one can obtain

$$
\begin{aligned}
\Xi= & -\left(\left(-v_{\rho}^{T} x\right) I-S\left(x_{0} v_{\rho}+S\left(v_{\rho}\right) x\right)\right) W_{\rho}\left(x_{0} v_{\rho}+S\left(v_{\rho}\right) x\right) \\
= & \lambda_{\rho} x_{0} v_{\rho}^{T} x v_{\rho}+v_{\rho}^{T} x W_{\rho} S\left(v_{\rho}\right) x+x_{0} S\left(v_{\rho}\right) W_{\rho} S\left(v_{\rho}\right) x \\
& +\left(S\left(v_{\rho}\right) S(x)-S(x) S\left(v_{\rho}\right)\right) W_{\rho}\left(x_{0} v_{\rho}+S\left(v_{\rho}\right) x\right)
\end{aligned}
$$

then, using the fact that $S^{2}\left(v_{\rho}\right)=v_{\rho} v_{\rho}^{T}-I, S\left(v_{\rho}\right) S(x)=x v_{\rho}^{T}-v_{\rho}^{T} x I$ and $W_{\rho} v_{\rho}=\lambda_{\rho} v_{\rho}$, one has $v_{\rho}^{T} x v_{\rho}=v_{\rho} v_{\rho}^{T} x=\left(S^{2}\left(v_{\rho}\right)+I\right) x, S\left(v_{\rho}\right) S(x) W_{\rho} S\left(v_{\rho}\right) x=-v_{\rho}^{T} x W_{\rho} S\left(v_{\rho}\right) x$ and $x_{0} S\left(v_{\rho}\right) S(x) W_{\rho} v_{\rho}=$ $-\lambda_{\rho} x_{0} S^{2}\left(v_{\rho}\right) x$. Finally, using all these identities and (31), (32), one can rewrite (24) function of the new variable as

$$
\left\{\begin{aligned}
\dot{\xi}= & -A_{d} \xi+B_{d}(X) \bar{\omega} \\
\dot{x}_{0}= & -\frac{1}{2} x^{T} \bar{\omega} \\
\dot{x}= & \frac{1}{2}\left(x_{0} I+S(x)\right) \bar{\omega}, \\
J_{d} \dot{\bar{\omega}}= & -B_{d}^{T}(X) \Gamma_{d} A_{d} \xi-S(\bar{\omega}) J_{d} \bar{\omega} \\
& +2\left(x_{0} I-S(x)\right)\left(\lambda_{\rho} I+S\left(v_{\rho}\right) W_{\rho} S\left(v_{\rho}\right)\right) x .
\end{aligned}\right.
$$

It is known that the tangent space of $\mathbb{S}^{3}$ at $\left[\begin{array}{l}1 \\ \mathbf{0}\end{array}\right]$ is given by the equation $y_{0}=0$ (with $\left.y=\left(y_{0}, y_{1}, y_{2}, y_{3}\right) \in \mathbb{R}^{4}\right)$ and the linearization of system (33) at $\Omega=(\xi, X, \bar{\omega})=\left(\mathbf{0}_{3 n},\left[\begin{array}{l}1 \\ \mathbf{0}\end{array}\right], \mathbf{0}\right)$ is given by

$$
\dot{Z}=\mathscr{A} Z \text {, with } \mathscr{A}=\left[\begin{array}{ccc}
-A_{d} & 0_{3 n \times 3} & H \\
0_{3 \times 3 n} & 0_{3} & I / 2 \\
-J_{d}^{-1} H^{T} \Gamma_{d} A_{d} & 2 J_{d}^{-1} G & 0_{3}
\end{array}\right] \text {, }
$$

where $Z=\left(z_{\xi}^{T}, z_{x}^{T}, z_{\omega}^{T}\right)^{T}$ with $z_{\xi}, z_{x}, z_{\omega}$ are the linearized vectors of $\xi, x, \bar{\omega}$, respectively. The matrices $G=\lambda_{\rho} I+S\left(v_{\rho}\right) W_{\rho} S\left(v_{\rho}\right)$ and $H=\left[\begin{array}{lll}H_{1}^{T} & \cdots & H_{n}^{T}\end{array}\right]^{T}$ with $H_{j}=S\left(\left(I+2 S^{2}\left(v_{\rho}\right)\right) R_{d} b_{j}^{d}\right)$.

Since $\Omega$ is not locally asymptotically stable, it is enough to show that $\mathscr{A}$ does not admit any eigenvalue with zero real part. Reasoning by contradiction, thus assume that $\mathscr{A}$ has an eigenvalue $i l, i^{2}=-1, l \geq 0$, with $Z^{l}=\left(z_{1}^{T}, z_{2}^{T}, z_{3}^{T}\right)^{T} \in \mathbb{C}^{3 n+6}$ a corresponding eigenvector. One gets the linear system of equations

$$
\left\{\begin{array}{rlrl}
-A_{d} z_{1}+ & H z_{3} & =i l z_{1}, \\
z_{3} / 2 & =i l z_{2}, \\
-J_{d}^{-1} H^{T} \Gamma_{d} A_{d} z_{1}+ & 2 J_{d}^{-1} G z_{2} & =i l z_{3} .
\end{array}\right.
$$

If $l=0$, one gets $z_{3}=z_{1}=0$ (since $A_{d}$ is positive definite) and $J_{d}^{-1} G z_{2}=0$. Recalling that $W_{\rho}$ is real symmetric with distinct eigenvalues, one can have that

$$
W_{\rho}=\lambda_{\rho} v_{\rho} v_{\rho}^{T}+\lambda_{1} v_{1} v_{1}^{T}+\lambda_{2} v_{2} v_{2}^{T}
$$


where $\left(v_{\rho}, v_{1}, v_{2}\right)$ is an orthonormal basis of $\mathbb{R}^{3}$ made of eigenvectors of $W_{\rho}$. By using the properties of $S\left(v_{\rho}\right)$, one gets

$$
G=\lambda_{\rho} v_{\rho} v_{\rho}^{T}+\left(\lambda_{\rho}-\lambda_{2}\right) v_{1} v_{1}^{T}+\left(\lambda_{\rho}-\lambda_{1}\right) v_{2} v_{2}^{T},
$$

implying that $\operatorname{det}(G)=\lambda_{\rho}\left(\lambda_{\rho}-\lambda_{1}\right)\left(\lambda_{\rho}-\lambda_{2}\right) \neq 0$ and thus $z_{2}=0$. Then the eigenvector $Z$ is equal to zero, which is impossible. Therefore, $l>0$ and one deduces that $z_{1}=\left(A_{d}+i l I_{3 n}\right)^{-1} H z_{3}, z_{2}=-\frac{i}{2 l} z_{3}$ and

$$
\left(i\left(J_{d} l+G / l\right)+H^{T} \Gamma_{d} A_{d}\left(A_{d}+i l I_{3 n}\right)^{-1} H\right) z_{3}=0 .
$$

One has $H^{T} \Gamma_{d} A_{d}\left(A_{d}+i l I_{3 n}\right)^{-1} H=\sum_{j=1}^{n}\left(R_{d}^{T} H_{j}\right)^{T} \Lambda_{j} A_{j}\left(A_{j}+i l I\right)^{-1}\left(R_{d}^{T} H_{j}\right)$. For $j=1, \ldots, n$, let $\left(v_{j 1}, v_{j 2}, v_{j 3}\right)$ be an orthonormal basis diagonalizing $\Lambda_{j}$ and $V$ the corresponding orthonormal matrix such that $\Lambda_{j}=V_{j} L_{j} V_{j}^{T}$, where $L_{j}=\operatorname{diag}\left(\lambda_{j 1}, \lambda_{j 2}, \lambda_{j 3}\right)$ with $\lambda_{j k} \in \mathbb{R}_{+}^{*}, j=1, \ldots, n, k=1,2,3$. Therefore, $P_{j}\left(\Lambda_{j}\right)=V_{j} P_{j}\left(L_{j}\right) V_{j}^{T}$, where $P_{j}\left(L_{j}\right)=\operatorname{diag}\left(P_{j}\left(\lambda_{j 1}\right), P_{j}\left(\lambda_{j 2}\right), P_{j}\left(\lambda_{j 3}\right)\right)$.

Recall that $A_{j}=P_{j}\left(\Lambda_{j}\right), j=1, \ldots, n$ where $P_{j}$ is a positive polynomial of degree two on $\mathbb{R}_{+}^{*}$, one deduces that

$$
\begin{aligned}
\Lambda_{j} A_{j}\left(A_{j}+i l I\right)^{-1} & =\Lambda_{j} P_{j}\left(\Lambda_{j}\right)\left(P_{j}\left(\Lambda_{j}\right)+i l I\right)^{-1} \\
& =V_{j} L_{j} P_{j}\left(L_{j}\right)\left(P_{j}\left(L_{j}\right)+i l I\right)^{-1} V_{j}^{T} \\
& =\sum_{k=1}^{3} \frac{\lambda_{j k} P_{j}\left(\lambda_{j k}\right)}{P_{j}\left(\lambda_{j k}\right)+i l} w_{j k} w_{j k}^{T},
\end{aligned}
$$

where we used the results on functions of matrices of Chapter 6 and section 6.2 in [39].

Multiplying Eq. (35) on the left by $\left(z_{3}^{*}\right)^{T}$ and using Eq. (36), yields

$$
i\left(z_{3}^{*}\right)^{T}\left(l J_{d}+G / l\right) z_{3}+\sum_{j=1}^{n} \sum_{k=1}^{3} \frac{\lambda_{j k} P_{j}\left(\lambda_{j k}\right)\left(P_{j}\left(\lambda_{j k}\right)-i l\right)}{P_{j}\left(\lambda_{j k}\right)^{2}+l^{2}}\left(\left(T_{j}^{*}\right)^{T} w_{j k}\right)\left(w_{j k}^{T} T_{j}\right)=0
$$

where $l>0, T_{j}=V_{j}^{T} R_{d}^{T} H_{j} z_{3}$ for $j=1, \ldots, n$ and $T_{j}^{*}$ is the conjugate of the complex vector $T_{j}$. Since $\left(z_{3}^{*}\right)^{T}\left(l J_{d}+G / l\right) z_{3}$ is a real number, one gets by taking the real part of Eq. (36)

$$
\sum_{j=1}^{n} \sum_{k=1}^{3} \frac{\lambda_{j k} P_{j}\left(\lambda_{j k}\right)^{2}}{P_{j}\left(\lambda_{j k}\right)^{2}+l^{2}}\left|w_{j k}^{T} T_{j}\right|^{2}=0
$$

where $|\cdot|$ denotes the mdulus of a complex number. One deduces at once that $T_{j}=H_{j} z_{3}=0$ for $j=1, \ldots, n$. Since two vectors $b_{j}^{d}$ are not colinear, one gets that $z_{3}=0$ and finally $Z=0$, which is again a contradiction.

If $\mathscr{A}$ does not have eigenvalues with positive real part, it would have only eigenvalues with negative real part and thus $\mathscr{A}$ would be Hurwitz, implying that (24) would be locally asymptotically stable with respect to $\Omega$. Since this is not true, $\mathscr{A}$ does admit at least one eigenvalue with positive real part. Thus, there exists an unstable manifold of dimension at least one in neighborhoods of the $\Omega_{j}^{ \pm}, j=2,3,4$, and since all trajectories converge to an equilibrium point, therefore (24) is almost globally asymptotically stable with respect to the two equilibrium points $\Omega_{1}^{ \pm}$. 


\section{REFERENCES}

1. Byrnes CI, Isidori A. On the attitude stabilization of rigid spacecraft. Automatica Jan. 1991; 27(1):87-95.

2. Wen JY, Kreutz-Delgado K. The attitude control problem. IEEE Transactions on Automatic Control Oct. 1991; 36(10): $1148-1162$.

3. Morin $\mathrm{P}$, Samson C. Time-varying exponential stabilization of a rigid spacecraft with two control torques. IEEE Transactions on Automatic Control Apr. 1997; 42(4):528-534.

4. Tayebi A, McGilvray S. Attitude stabilization of a VTOL quadrotor aircraft. IEEE Transactions On Control Systems Technology May. 2006; 14(3):562-571.

5. Mayhew CG, Sanfelice RG, Teel AR. Robust global asymptotic attitude stabilization of a rigid body by quaternionbased hybrid feedback. Proceedings of the Joint 48th IEEE Conference on Decision and Control and 28th Chinese Control Conference (CDC-CCC), Shanghai, Dec. 2009; 2522-2527.

6. Rifai H, Guerrero-Castellanos JF, Marchand N, Poulin-Vittrant G. Biomimetic-based output feedback for attitude stabilization of a Flapping-wing Micro Aerial Vehicle. Robotica Apr. 2013; 31(6):955-968.

7. Rifai H, Guerrero-Castellanos JF, Marchand N, Poulin-Vittrant G. Bounded attitude control of a Flappingwing Micro Aerial Vehicle using direct sensors measurements. IEEE International Conference on Robotics \& Automation (ICRA), Kobe, 2009; 3644-3650.

8. Bach R, Paielli R. Linearization of attitude-control error dynamics. IEEE Transactions on Automatic Control Oct. 1993; 38(10):1521-1525.

9. Tsiotras P. Stabilization and optimality results for the attitude control problem. Journal of Guidance, Control, and Dynamics July-Aug. 1996; 19(04):772-779.

10. Bayadi R, Banavar RN. Almost global attitude stabilization of a rigid body for both internal and external actuation schemes. European Journal of Control Jan. 2014; 20(1):45-54.

11. Chaturvedi N, Sanyal A, McClamroch N. Rigid-body attitude control. IEEE Control Systems Magazine Jun. 2011; 31(3):30-51.

12. Lizarralde F, Wen J. Attitude control without angular velocity measurement: a passivity approach. IEEE Transactions on Automatic Control Mar. 1996; 41(3):468-472.

13. Tsiotras P. Further passivity results for the attitude control problem. IEEE Transactions on Automatic Control Nov. 1998; 43(11):1597-1600.

14. Akella MR. Rigid body attitude tracking without angular velocity feedback. Systems \& Control Letters Apr. 2001; 42(4):321-326.

15. Costic BT, Dawson DM, Queiroz MSd, Kapila V. Quaternion-based adaptive attitude tracking controller without velocity measurements. Journal of Guidance, Control, and Dynamics Nov. 2001; 24(6):1214-1222.

16. Tayebi A. Unit quaternion-based output feedback for the attitude tracking problem. IEEE Transactions on Automatic Control Jul. 2008; 53(6):1516-1520.

17. Schlanbusch R, Grøtli EI, Loria A, Nicklasson PJ. Hybrid attitude tracking of rigid bodies without angular velocity measurement. Systems \& Control Letters Apr. 2012; 61(4):595-601.

18. Xiao B, Hu Q, Shi P. Attitude stabilization of spacecrafts under actuator saturation and partial loss of control effectiveness. IEEE Transactions On Control Systems Technology Nov. 2013; 21(6):2251-2263.

19. Thakur D. Adaptation, gyro-free stabilization, and smooth angular velocity observers for attitude tracking control applications. PhD Thesis, The University of Texas at Austin Aug. 2014.

20. Tanygin S, Shuster M. The many triad algorithms. AAS/AIAA, 2007; 81-99.

21. Batista P, Silvestre C, Oliveira P. Sensor-based globally asymptotically stable filters for attitude estimation: Analysis, design, and performance evaluation. IEEE Transactions on Automatic Control Aug. 2012; 57(8):20952100.

22. Zamani M, Trumpf J, Mahony R. Nonlinear attitude filtering: A comparison study. arXiv:1502.03990 [cs.SY] Feb. 2015; .

23. Crassidis J, Markley F, Cheng F. Survey of nonlinear attitude estimation methods. Journal of guidance, control, and dynamics Jan. 2007; 30(1):12-28.

24. Mahony R, Hamel T, Pflimlin JM. Nonlinear complementary filters on the Special Orthogonal group. IEEE Transactions on Automatic Control Jun. 2008; 53(5):1203-1218.

25. Crassidis JL, Junkins JL. Optimal Estimation of Dynamic Systems. 2nd edn., Chapman \& Hall / CRC Applied Mathematics \& Nonlinear Science, 2012.

26. Tayebi A, Roberts A, Benallegue A. Inertial vector measurements based velocity-free attitude stabilization. IEEE Transactions on Automatic Control Nov. 2013; 58(11):2893-2898.

27. Bhat SP, Bernstein DS. A topological obstruction to continuous global stabilization of rotational motion and the unwinding phenomenon. Systems \& Control Letters Jan. 2000; 39(1):63-70. 
28. Benziane L, Benallegue A, Tayebi A. Attitude stabilization without angular velocity measurements. IEEE International Conference on Robotics \& Automation (ICRA), Hong Kong, China, 2014; 3116-3121.

29. Hatcher A. Algebraic Topology. Cambridge University Press, 2002.

30. Munkres JR. Topology. 2nd edn., Prentice Hall, 2000.

31. Markley FL, Crassidis JL. Fundamentals of Spacecraft Attitude Determination and Control. Microcosm Press and Springer, 2014.

32. Smith L. Linear Algebra. 3rd edn., 1998.

33. Waerden BLvd. Algebra. Frederick Ungar Publishing Co., New York, 1970.

34. Hartshorne R. Algebraic Geometry. Springer, 1977.

35. Thakur D, Akella MR, Mazenc F. Partial lyapunov strictification: Smooth angular velocity observers for attitude tracking control. Journal of Guidance, Control, and Dynamics 2015; 38(3):442-451.

36. Dorf RC, Bishop RH. Modern Control Systems. Twelfth edn., Prentice Hall, 2011.

37. MathWorks. How globalsearch and multistart work, http://fr.mathworks.com/help/gads/how-globalsearch-andmultistart-work.html April 2015.

38. Bullo F, Lewis A. Geometric Control of Mechanical Systems : modeling, analysis, and design for simple mechanical control systems. Springer, 2005.

39. Horn RA, Johnson CR. Topics in Matrix Analysis. Cambridge University Press, 1991. 


\begin{tabular}{|c|c|c|}
\hline gains & $l(\kappa(m))$ & $u(\kappa(m))$ \\
\hline \hline$\rho_{i}(i=1,2)$ & 4 & 17 \\
\hline$a_{i 0}(i=1,2)$ & 0.1 & 0.5 \\
\hline$a_{i 1}(i=1,2)$ & 0.001 & 0.05 \\
\hline$a_{i 2}(i=1,2)$ & 0.00001 & 0.005 \\
\hline$\gamma_{i j}(i=1,2, j=1,2,3)$ & 20 & 120 \\
\hline
\end{tabular}

Table I. Lower and upper limits

\begin{tabular}{|c|c|}
\hline gains & ISE $g_{\text {ise }}(\kappa)=\int_{0}^{\infty}\left(\|\bar{q}\|^{2}+0.1\|\tau\|^{2}\right) d t$ \\
\hline \hline$\rho_{i}(i=1,2)$ & {$[9.0339,7.3266]$} \\
\hline$a_{1(j-1)}(j=1,2,3)$ & {$[0.4061,0.0365,0.0034]$} \\
\hline$a_{2(j-1)}(j=1,2,3)$ & {$[0.2898,0.0205,0.0027]$} \\
\hline$\gamma_{1 j}(j=1,2,3)$ & {$[30.7484104 .416593 .6847]$} \\
\hline$\gamma_{2 j}(j=1,2,3)$ & {$[93.4728,20,106.8129]$} \\
\hline
\end{tabular}

Table II. Selected optimal gain values

\begin{tabular}{|c|c|}
\hline parameters & values calculated with ISE criterion \\
\hline \hline$\Lambda_{1}$ & $\operatorname{diag}([30.7484104 .4165$ 93.6847] $)$ \\
\hline$\Lambda_{2}$ & $\operatorname{diag}([93.4728,20,106.8129])$ \\
\hline$A_{1}$ & $\operatorname{diag}([4.743041 .286833 .6668])$ \\
\hline$A_{2}$ & $\operatorname{diag}([25.79631 .779833 .2838])$ \\
\hline$W_{\rho}$ & {$\left[\begin{array}{ccc}14.9750 & -0.0025 & -2.8686 \\
-0.0025 & 16.3601 & -0.0053 \\
-2.8686 & -0.0053 & 1.3851\end{array}\right]$} \\
\hline eigenvectors & $v_{\rho 1}= \pm\left[\begin{array}{c}0 \\
-1 \\
0\end{array}\right]$ \\
of $W_{\rho}$ & $\left.\begin{array}{c}0.9801 \\
0.0018 \\
-0.1984\end{array}\right], v_{\rho 3}= \pm\left[\begin{array}{l}-0.1984 \\
-0.0004 \\
v_{\rho 2}= \pm .9801\end{array}\right]$ \\
\hline $\begin{array}{c}\text { eigenvalues } \\
\text { of } W_{\rho}\end{array}$ & $\lambda_{\rho 1}=16.3601, \lambda_{\rho 2}=15.5558$ \\
$\lambda_{\rho 3}=0.8044$
\end{tabular}

Table III. Gain matrices 